\title{
Neuroscience of morality and teacher education
}

Hyemin Han

University of Alabama, Tuscaloosa AL, United States

hyemin.han@ua.edu

\section{Introduction ${ }^{1}$}

Given that teachers become primary fundamental exemplars and models for their students and the students are likely to emulate the presented teachers' behaviors, it is necessary to consider how to promote teachers' abilities as potential moral educators during the course of teacher education. To achieve this ultimate aim in teacher education, as argued by moral philosophers, psychologists, and educators, teachers should be able to well understand the mechanisms of moral functioning and how to effectively promote moral development based on evidence. Recent findings in the fields of social sciences that have examined how morality is functioning among human beings, moral psychology in particular, provide us with the evidence through experiments and observations. Furthermore, thanks to the development of scientific research methods that enable researchers to investigate the neural-level nature of human behavior, the researchers now can examine neural correlates of moral functioning that constitutes the basis of moral behavior and development. To facilitate the understanding of the field of neuroscience of morality and its educational implications within the context of teacher education, first, the recent trend in the field will be reviewed, and second, how it can provide useful insights to teachers and teacher educations will be discussed.

\section{Neuroscience of morality}

Recent studies in the field of neuroscience have provided empirical findings regarding diverse aspects of human morality including, but not limited to, moral cognition, emotion, intuition, motivation, behavior, and development. They have discovered not only brain regions associated with the aforementioned aspects of moral functioning, but also how different neural circuitries consisting of the brain regions are interacting with each other. For instance, various functional neuroimaging studies that used moral dilemmas as experimental paradigms have demonstrated that regions associated with cognition were mainly involved in utilitarian decision making while those associated with emotion were more likely to be activated during deontological decision making (see Han (2017) for quantitative review). In addition to this kind of brain localization studies, Han, Chen, Jeong, \& Glover's (2016) study that focused on interactions between neural circuitries reported that the neural network associated with self-related psychological processes, such as autobiographical memory processing, significantly interacted with morality-related brain. In addition to the prior moral judgment neuroimaging studies that have examined the neurallevel mechanisms associated with moral functioning by presenting participants with different types of moral dilemmas, a recent structural neuroimaging study investigated the relationship between brain structures and the development of moral reasoning among participants (Prehn et al., 2015). This study reported that participants' medial prefrontal cortex gray matter thickness was positively correlated with their more sophisticated post-conventional moral reasoning.

' To appear in Peters, M. A., (in preparation). Encyclopedia of Teacher Education. Singapore: Springer. 
In addition to the neuroimaging studies, there have been recent neurostimulation studies that attempted to manipulate moral psychological processes among human participants. Neuroimaging studies were not able to provide any information regarding a causal relationship among brain regions and specific psychological functionalities because used neuroimaging methods were correlational, not causal; they were only able to examine correlation or association. On the other hand, neurostimulation methods, such as the transcranial magnetic stimulation (TMS) and transcranial direct current stimulation (tDCS), can alter neural activity in target regions, so they can enable researchers to examine the causal relationship. For instance, Young, Camprodon, Hauser, Pascual-Leone, and Saxe (2010) showed that the TMS on the temporoparietal junction can alter participants' perception on others' moral belief. In addition, Ruff, Ugazio, and Fehr (2013) reported that the tDCS on the prefrontal cortex increased or decreased socio-moral rule conformity. These results identified a causal relationship between the target region and target psychological functionality in addition to a correlational relationship by demonstrating activity in the target region altered psychological and behavioral outcomes.

As reviewed above, advances in neuroscience have allowed researchers to investigate the underlying biological and neural mechanisms of human moral functioning. Without the developments of neuroscientific research methods, moral psychological processes, such as the mechanisms of the involvements of cognition and emotion in moral judgment and the association between self-related processes and moral functioning (Han, 2017; Han et al., 2016), could not be well examined with scientific evidence. Furthermore, recent research on neurostimulation has demonstrated that how researchers can alter moral psychological processes, such as the perception of moral belief (Young et al., 2010) and judgment on socio-moral rules (Ruff et al., 2013). Given these, educators may consider how to learn from neuroscience of morality to improve education in practice, particularly that addresses socio-moral development among students.

\section{Several debates regarding the application of neuroscience in education}

Although neuroscience of morality has illuminated many aspects of human moral functioning that could not be studied without newly developed technologies, how to apply findings from neuroscience research in educational settings would be a difficult question to answer. From the perspectives of teachers who might not have sufficient background knowledge in neuroscience, findings from functional neuroimaging experiments per se might not be able to provide them with concrete educational implications. For example, a fact that brain regions associated with emotion are significantly activated when a person is making deontological moral judgment does not say anything about what kind of educational approaches should be utilized to effectively promote students' moral reasoning. If a neuroimaging study reported that the gray matter volume in the prefrontal cortex is associated with more sophisticated moral reasoning (Prehn et al., 2015), should teachers utilize invasive neurosurgical or neurostimulation methods to increase the regional gray matter volume to promote moral development? The answer to this question might not be "yes." Hence, it is required to carefully consider how to learn from neuroscience to improve educational practices in real educational settings. 
Moreover, a mere understanding of neuroscience and trial to apply it in educational activities could also be misleading. A recent survey study showed that such an issue existing among school teachers in general (Howard-Jones, 2014). Howard-Jones (2014) surveyed some misconceptions regarding the relationship between neuroscience and education. For example, the localization of psychological functionalities in human brains was pervasively accepted by school teachers despite findings from recent neuroimaging studies focusing on the interactions among diverse neural circuitries (e.g., Han et al., 2016). As a result, many of the surveyed teachers believed that it makes sense to focus on a specific modality of instructional method given that a specific intellectual functionality is associated with a specific brain region although recent neuroimaging findings suggested that such a localization is not the only way to understand neural-level processes. Hence, it would be necessary to promote accurate understandings regarding how human brains work within the context of moral functioning while avoiding possible misconceptions among teachers.

In addition, there have been debates about the utility of educational neuroscience as a field that integrates neuroscience and education to improve education in practice. Bowers (2016) argued that neuroscientific evidence has not added something brand new to the existing literature of educational research. According to his argument, the majority of scientific and practical contributions in educational research has been made by non-neuroscientific psychological studies, and findings from neuroscientific studies have been somehow redundant and could not directly contribute to education in practice. Instead, he argued that psychological intervention studies that are based on traditional non-neuroscientific psychological research can provide educators with useful insights more directly. Given the issues related to the application of neuroscience in education, Bowers's criticism on educational neuroscience is informative while considering how to learn from neuroscience to improve education. As discussed above, it would not be possible for educators to attempt to manipulate students' neural activity based on brain localization and neurostimulation studies to promote their moral and intellectual development. If this is the case, then as Bowers criticized, ideas from traditional psychological intervention experiments, which have been contributed to the development of psychological interventions in the reality, might be able to provide educators with more practical insights about how to improve their educational activities in classrooms.

\section{Rethink how to learn from neuroscience of morality to improve education in practice and teacher education}

Given these issues and criticisms, it is necessary to consider how to properly organize the framework of the application of neuroscience within the context of education in practice and teacher education. To overcome the issues associated the application of neuroscience in education, the neuromyths among teachers in particular, reviewing discussions regarding how to fill the gap between educational research, education in practice, and teacher education can be informative. A framework proposed by Wortham (2018) might provide some points to be considered related to this point. Wortham (2018) argued that trials to merely and directly implement findings from educational research in education in practice could not be successful unlike the case of the implementation of evidence-based techniques in several other fields, such as medicine. Instead, it is necessary to share and understand repertories across researchers and educators. Moreover, Immordino-Yang and Damasio (2007) proposed that research on 
neuroscience of morality, particularly that focusing on the role of emotion and cognition, can provide educators with useful and concrete ideas about how to constrain possibilities and set hypotheses and directions while developing educational activities.

Hence, instead of pursuing the direct implementation of neuroscience of morality in education in practice, educators may need to consider how to get useful heuristics from neuroscience to enhance their educational activities. In addition, teacher educators who intend to introduce neuroscience to educators should carefully consider how to provide the educators with such heuristics instead of merely referring to findings in neuroscience. By doing so would be a possible way to share useful ideas proposed by Wortham (2018), the expansion of repertories across neuroscience researchers and educators, and Immordino-Yang and Damasio (2007), learning from neuroscience to set new hypotheses and directions in the development of educational activities, with educators who are interested in the application of neuroscience of morality in their educational activities during the course of teacher education.

Teacher educators may be interested in understanding how such a framework can work in the reality with a concrete example in the field in order to provide educators with practical information. Here is one concrete example that demonstrates how neuroscience of morality can provide moral education with useful heuristics about how to develop a more effective educational program. Han, Kim, Jeong, and Cohen's (2017) moral education program that utilized the stories of moral exemplars was initially inspired by studies in neuroscience of morality that examined the involvement of self-related processes in the moderation of moral emotion and motivation. A meta-analysis of previous moral psychological neuroimaging studies (Han, 2017) and a functional neuroimaging experiment (Han et al., 2016) showed significant associations among self-related brain regions and morality-related brain regions. Based on these findings, Han et al. (2017) designed and tested an exemplar-applied moral education program that used close-other exemplars (e.g., friends, family members) that might have stronger psychological connectivity with students; their study reported that the aforementioned program inspired by neuroscience significantly promoted students' moral motivation. Han et al. (2017) attempted to directly focus on activity in specific brain regions after reviewing localization studies. Instead, they tried to get more practical insights (e.g., ideas regarding the association between self and morality) from neuroscience research and were able to produce concrete educational outcomes. This example presents educators with how to obtain heuristics from neuroscience while developing educational activities.

\section{Conclusion}

Neuroscience of morality has contributed to the expansion of the understanding of the neurallevel mechanisms of moral psychological processes with scientific evidence. It has enabled researchers to investigate psychological processes underlying moral functioning with neuroimaging methods and alter such processes with neurostimulation methods. Although it is obvious that the contributions of neuroscience of morality to the field are valuable, how to apply neuroscience of morality in education in practice and teacher education could be a difficult question to answer given criticisms regarding neuromyths among teachers and utility of educational neuroscience. To address this issue, some discussions regarding how to fill the gap between educational research and education in practice have been reviewed with a concrete 
example showing how educators can learn from findings in neuroscience to improve moral education. As reviewed, trials to understand and share repertories across different realms and to get useful heuristics from neuroscience of morality can be more successful compared with mere implementations of such findings. Teacher education programs that intend to employ neuroscience as a component may also need to consider these points to avoid the issue of neuromyths and to help teachers learn how to appropriately develop their educational activities based on neuroscience.

\section{Reference}

Bowers, J. S. (2016). Psychology, not educational neuroscience, is the way forward for improving educational outcomes for all children: Reply to Gabrieli (2016) and HowardJones et al. (2016). Psychological Review, 123(5), 628-635. http://doi.org/10.1037/rev0000043

Han, H. (2017). Neural correlates of moral sensitivity and moral judgment associated with brain circuitries of selfhood: A meta-analysis. Journal of Moral Education, 46(2), 97-113. http://doi.org/10.1080/03057240.2016.1262834

Han, H., Chen, J., Jeong, C., \& Glover, G. H. (2016). Influence of the cortical midline structures on moral emotion and motivation in moral decision-making. Behavioural Brain Research, 302, 237-251. http://doi.org/10.1016/j.bbr.2016.01.001

Han, H., Kim, J., Jeong, C., \& Cohen, G. L. (2017). Attainable and Relevant Moral Exemplars Are More Effective than Extraordinary Exemplars in Promoting Voluntary Service Engagement. Frontiers in Psychology, 8, 283. http://doi.org/10.3389/fpsyg.2017.00283

Howard-Jones, P. a. (2014). Neuroscience and education: myths and messages. Nature Reviews. Neuroscience, 15(12). http://doi.org/10.1038/nrn3817

Immordino-Yang, M. H., \& Damasio, A. (2007). We Feel, Therefore We Learn: The Relevance of Affective and Social Neuroscience to Education. Mind Brain and Education, 1(1), 3-10. http://doi.org/10.1111/j.1751-228X.2007.00004.X

Prehn, K., Korczykowski, M., Rao, H., Fang, Z., Detre, J. A., \& Robertson, D. C. (2015). Neural Correlates of Post-Conventional Moral Reasoning: A Voxel-Based Morphometry Study. PLOS ONE, 10(6), e0122914. http://doi.org/10.1371/journal.pone.0122914

Ruff, C. C., Ugazio, G., \& Fehr, E. (2013). Changing social norm compliance with noninvasive brain stimulation. Science (New York, N.Y.), 342, 482-4. http://doi.org/10.1126/science.1241399

Wortham, S. (2018). Reimagining Research and Practice in Education. In D. W. Kritt (Ed.), Constructivist Education in an Age of Accountability (pp. 317-326). Cham, Switzerland: Springer International Publishing. http://doi.org/10.1007/978-3-319-66050-9_16

Young, L., Camprodon, J. A., Hauser, M., Pascual-Leone, A., \& Saxe, R. (2010). Disruption of the right temporoparietal junction with transcranial magnetic stimulation reduces the role of beliefs in moral judgments. Proceedings of the National Academy of Sciences of the United States of America, 107, 6753-6758. http://doi.org/10.1073/pnas.0914826107 